\title{
NEW RECORD OF Coomansus venezolanus (Loof, 1964) \\ Jairajpuri \& Khan, 1977 (Mononchida: Mononchidae) FOR VIETNAM WITH FIRST DESCRIPTION OF A MALE
}

\author{
Vu Thi Thanh Tam \\ Institute of Ecology and Biological Resources, VAST
}

\begin{abstract}
Coomansus venezolanus (Loof, 1964) Jairajpuri \& Khan, 1977 from Cao Loc, Lang Son province is described and illustrated and represents a new record for Vietnam. The species is characterized by its body size, 1.9-2.1 mm long, a strongly sclerotized buccal cavity with apex of dorsal tooth situated at 26-30\% of buccal cavity length from anterior end and vulva at $60-64 \%$ of body length from anterior end. A single male specimen is reported for the first time and is characterized by having fifteen spaced ventromedian precloacal supplements, a $74 \mu \mathrm{m}$ long spicule; well developed lateral accessory pieces, $12.5 \mu \mathrm{m}$ long and a weakly developed gubernaculum, $11.4 \mu \mathrm{m}$ long. Measurements and description of the Vietnamese female specimens fit well the holotype, except for a slightly slenderer body $(\mathrm{a}=27-31 v s 21)$ and shorter tail $(\mathrm{c}=20$ 28 vs 18); they also agree with the specimen from Himachal Pradesh, India apart from a slightly shorter tail and a slightly stouter body.
\end{abstract}

Keywords: Mononchidae, Coomansus, new record, Lang Son, Vietnam.

Citation: Vu Thi Thanh Tam, 2016. New record of Coomansus venezolanus (Loof, 1964) Jairajpuri \& Khan, 1977 (Mononchida: Mononchidae) for Vietnam with first description of a male. Tap chi Sinh hoc, 38(3): 293-297. DOI: 10.15625/0866-7160/v38n3.8023.

*Corresponding author: vtam7572@yahoo.com

\section{INTRODUCTION}

Coomansus venezolanus (Loof, 1964) Jairajpuri \& Khan, 1977 was originally described as Mononchus venezolanus (Mononchidae) by Loof (1964) [4] from only one female collected together with a juvenile from soil around roots of garlic at 1500-1800 m altitude, from El Jarillo, Venezuela. The main characters were: (i) a strong massive dorsal tooth with its tip located in the anterior third of the buccal cavity; and (ii) absence of 'ventral' ridge in the buccal cavity. Jairajpuri \& Khan (1977) [2] proposed the new genus Coomansus Jairajpuri \& Khan, 1977 (Mononchidae), based on the following main characters: (i) lip and labial papillae well developed; (ii) the absence of the ventral ridge in the buccal cavity, and transferred two species of Mononchus, M. campbelli Allgen, 1929 and M. venezolanus Loof, 1964, to the new genus Coomansus. Jairajpuri \& Khan, 1982 recorded Coomansus venezolanus from Himachal Pradesh, India based on a female with a slightly slenderer body than in the holotype [3].
In Vietnam, up to now, studies on predaceous nematodes of the order Mononchida have revealed 63 species, which belong to ten genera of four families [5, 8, 9]. Currently, only one species of the genus Coomansus, C. parvus has been reported in Vietnam [5]. In the present paper, C. venezolanus was identified from Cao Loc, Lang Son province, Vietnam, representing a new record for the nematode fauna in Vietnam. For the first time, a male specimen was found and described.

\section{MATERIALS AND METHODS}

Soil samples were collected from natural forest areas in Cao Loc, Lang Son province, Vietnam. Nematodes were extracted from soil sample by modified Baermann funnel technique (Southey, 1986) [7], killed by heat, fixed in formaldehyde 4\%, transferred to anhydrous glycerol according to Seinhorst (1959) [6], and mounted on glass slides for microscopic observation. Permanent slides were stored at the Department of Nematology, IEBR. Figures were drawn using an Olympus microscope 
CH40 with drawing tube and illustrations were edited by Adobe Illustrator CS6. Photographs were taken with an Olympus DP digital camera on an Olympus microscope BX51 and edited by Adobe Photoshop CS6.

IEBR stands for Institute of Ecology and Biological Resources; VAST stands for Vietnam Academy of Science and Technology.

\section{RESULTS AND DISCUSSION}

\section{Coomansus venezolanus (Loof, 1964) Jairajpuri and Khan, 1977 (Fig. 1)}

Material: Five females and 1 male in good condition. Measurements: See table 1.

\section{Description:}

Female: Moderately stout nematodes of medium size, 1.8-2.1 mm long. Habitus after fixation ventrally arcuate, especially towards posterior end. Cuticle smooth under light microscope, $3 \mu \mathrm{m}$ thick throughout body. Lip region offset from body contour, 35-38 $\mu \mathrm{m}$ width and 12-14 $\mu \mathrm{m}$ height. Labial papillae prominent, protruded. Amphideal fovea cupshaped, aperture $4 \mu \mathrm{m}$, situated at 16-18 $\mu \mathrm{m}$ from anterior end of body or at the level of apex of dorsal tooth. Buccal cavity medium size 40.5-41.5 $\mu \mathrm{m}$ long and 19.4-20.4 $\mu \mathrm{m}$ wide, strongly sclerotized. Apex of dorsal tooth sharp, pointing forward, located at 26-30\% of buccal cavity length from anterior end. No weak longitudinal ridges observed in ventrosublateral walls of buccal cavity. Oesophagus cylindroid, 473-473 $\mu \mathrm{m}$ long, nerve ring at about one-third of its length from anterior end; secretory-excretory pore located just posterior to nerve ring. Oesophago-intestinal junction non-tuberculate. Rectum straight, thick-walled and muscular, 31.5-34 $\mu \mathrm{m}$. Reproductive system didelphic-amphidelphic, both branches equally developed with ovary reflexed. Sphincter absent between oviduct and uterus. Vulva, a transverse slit in ventral view, vagina short with par refringens vaginae sclerotized and visible as 2 pieces in optical section. Tail bent ventrally, 92-101 $\mu \mathrm{m}$ long, conoid, tapering regularly to narrowly rounded terminus. Caudal glands rudimentary and spinneret absent.
Male: Similar to female in general morphology and size, $1.8 \mathrm{~mm}$ long. Buccal cavity $39.6 \mu \mathrm{m}$ long and $19.4 \mu \mathrm{m}$ wide, strongly sclerotized. Apex of dorsal tooth located at $27 \%$ of buccal cavity length from anterior end. Oesophagus cylindroid, $436 \mu \mathrm{m}$ long. Rectum and pre-rectum indistinct. Tail similar to female but relatively shorter and with more rounded terminus, $85.5 \mu \mathrm{m}$ long. Caudal glands rudimentary and terminal opening absent. Fifteen spaced ventromedian precloacal supplements present. Testes paired, opposed, outstretched. Spicules paired, ventrally arcuate, $74 \mu \mathrm{m}$ long and $10 \mu \mathrm{m}$ width. Lateral accessory piece well developed with bifurcated tip, $12.5 \mu \mathrm{m}$ long. Gubernaculum weakly developed, $11.4 \mu \mathrm{m}$.

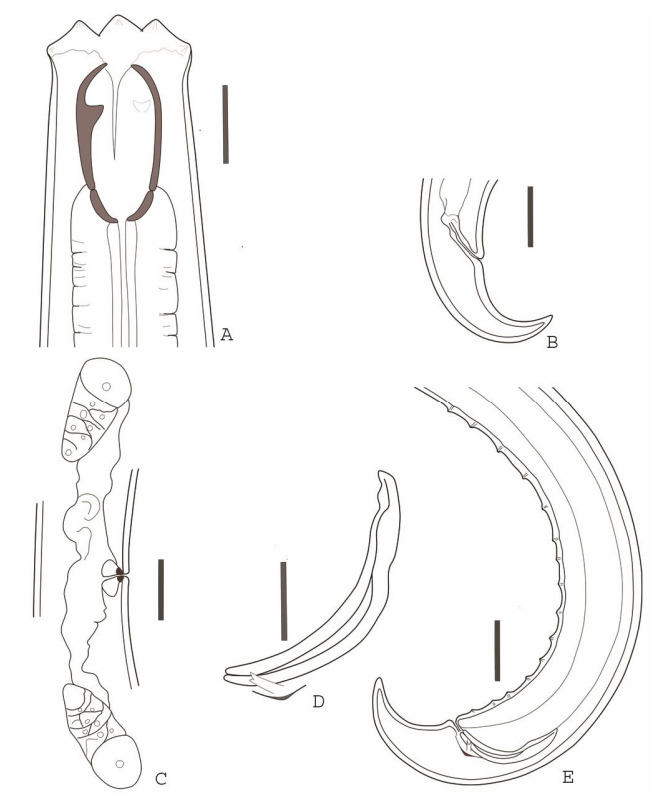

Figure 1. Coomansus venezolanus (Loof, 1964) Jairajpuri \& Khan, 1977

A. Female anterior region; B. Female tail; C. Female genital system; D. Spicule, gubernaculum and lateral accessory piece; E. Male tail region. Scale bars: A, $\mathrm{D}=20 \mu \mathrm{m} ; \mathrm{B}, \mathrm{C}, \mathrm{E}=40 \mu \mathrm{m}$.

Locality: Cao Loc (Lang Son province, Vietnam).

Remarks: The measurements and description of Vietnamese specimens correspond well to the holotype from Venezuela (Loof, 1964) [4] and a female specimen from 
Himachal Pradesh, India (Jairajpuri and Khan, 1.62) vs 21) and shorter tail (c = 20.5-28.7 (22.4 1982) [3], except for slightly slenderer body \pm 3.56 ) vs 18-19). A male specimen is here compared with the holotype $(\mathrm{a}=27-31(28.2 \pm$ described for the first time.

Table 1. Morphometric data of Coomansus venezolanus (Loof, 1964) Jairajpuri and Khan, 1977

\begin{tabular}{|c|c|c|c|c|}
\hline & \multicolumn{4}{|c|}{ Coomansus venezolanus } \\
\hline Locality & $\begin{array}{l}\text { Type El } \\
\text { Jarillo, } \\
\text { Venezuela }\end{array}$ & $\begin{array}{l}\text { Himachal } \\
\text { Pradesh, } \\
\text { India } \\
\end{array}$ & \multicolumn{2}{|c|}{ Lang Son, Vietnam } \\
\hline & Loof, 1964 & $\begin{array}{l}\text { Jairajpuri \& } \\
\text { Khan, } 1982 \\
\end{array}$ & \multicolumn{2}{|c|}{ Present paper } \\
\hline $\mathrm{n}$ & Holotype + & 1 ㅇ & 5 우우 & 10 \\
\hline $\mathrm{L}(\mu \mathrm{m})$ & 2200 & 2550 & $\begin{array}{c}1918-2036 \\
(1952 \pm 47.38)\end{array}$ & 1.8 \\
\hline $\mathrm{a}$ & 21 & 34 & $\begin{array}{c}27-31 \\
(28.2 \pm 1.62)\end{array}$ & 32 \\
\hline $\mathrm{b}$ & 4.2 & 4.5 & $\begin{array}{c}4.1-4.4 \\
(4.2 \pm 0.1)\end{array}$ & 4.1 \\
\hline $\mathrm{c}$ & 18 & 19 & $\begin{array}{c}20.2-28.7 \\
(22.4 \pm 3.56)\end{array}$ & 21.2 \\
\hline$c^{\prime}$ & $2.7^{\mathrm{a}}$ & 3 & $\begin{array}{c}1.7-2.7 \\
(2.3 \pm 0.38)\end{array}$ & 2.1 \\
\hline $\mathrm{V}(\%)$ & 60 & 61 & $\begin{array}{c}60.7-64 \\
(62 \pm 1.21)\end{array}$ & - \\
\hline Buccal cavity length $(\mu \mathrm{m})$ & 42 & 38 & $\begin{array}{c}40.5-41.4 \\
(41.2 \pm 0.45)\end{array}$ & 40 \\
\hline Buccal cavity width $(\mu \mathrm{m})$ & 18 & 16 & $\begin{array}{c}19.4-20.3 \\
(20 \pm 0.4)\end{array}$ & 19.4 \\
\hline $\begin{array}{l}\text { Apex of dorsal tooth position } \\
\text { from anterior end of buccal } \\
\text { cavity }(\%)\end{array}$ & $25^{\mathrm{a}}$ & 20 & $\begin{array}{c}26-30 \\
(27.2 \pm 1.79)\end{array}$ & 27 \\
\hline Lip width $(\mu \mathrm{m})$ & $36.8^{\mathrm{a}}$ & 37 & $\begin{array}{c}35-37.5 \\
(36 \pm 1.34)\end{array}$ & 34.3 \\
\hline Lip height $(\mu \mathrm{m})$ & 12 & 16 & $\begin{array}{c}12.3-14 \\
(13 \pm 0.72)\end{array}$ & 13.2 \\
\hline Neck length $(\mu \mathrm{m})$ & 524 & 567 & $\begin{array}{l}473-473 \\
(473 \pm 0)\end{array}$ & 436 \\
\hline Body diameter at vulva $(\mu \mathrm{m})$ & $87.5^{\mathrm{a}}$ & $75^{\mathrm{a}}$ & $\begin{array}{c}62.5-75.7 \\
(69.8 \pm 4.82)\end{array}$ & 56.3 \\
\hline Anal body diameter $(\mu \mathrm{m})$ & $44.7^{\mathrm{a}}$ & $44^{\mathrm{a}}$ & $\begin{array}{c}37.5-40 \\
(38.2 \pm 0.95)\end{array}$ & 40.7 \\
\hline Tail length $(\mu \mathrm{m})$ & $122^{\mathrm{a}}$ & 130 & $\begin{array}{c}67.5-101 \\
(98.1 \pm 12.73)\end{array}$ & 85.5 \\
\hline Spicule length $(\mu \mathrm{m})$ & - & - & - & 74 \\
\hline Lateral accessory piece $(\mu \mathrm{m})$ & - & - & - & 12.5 \\
\hline Gubernaculum $(\mu \mathrm{m})$ & - & - & - & 11.4 \\
\hline Supplements & - & - & - & 15 \\
\hline
\end{tabular}

${ }^{a}$ calculated from original figure and description. 


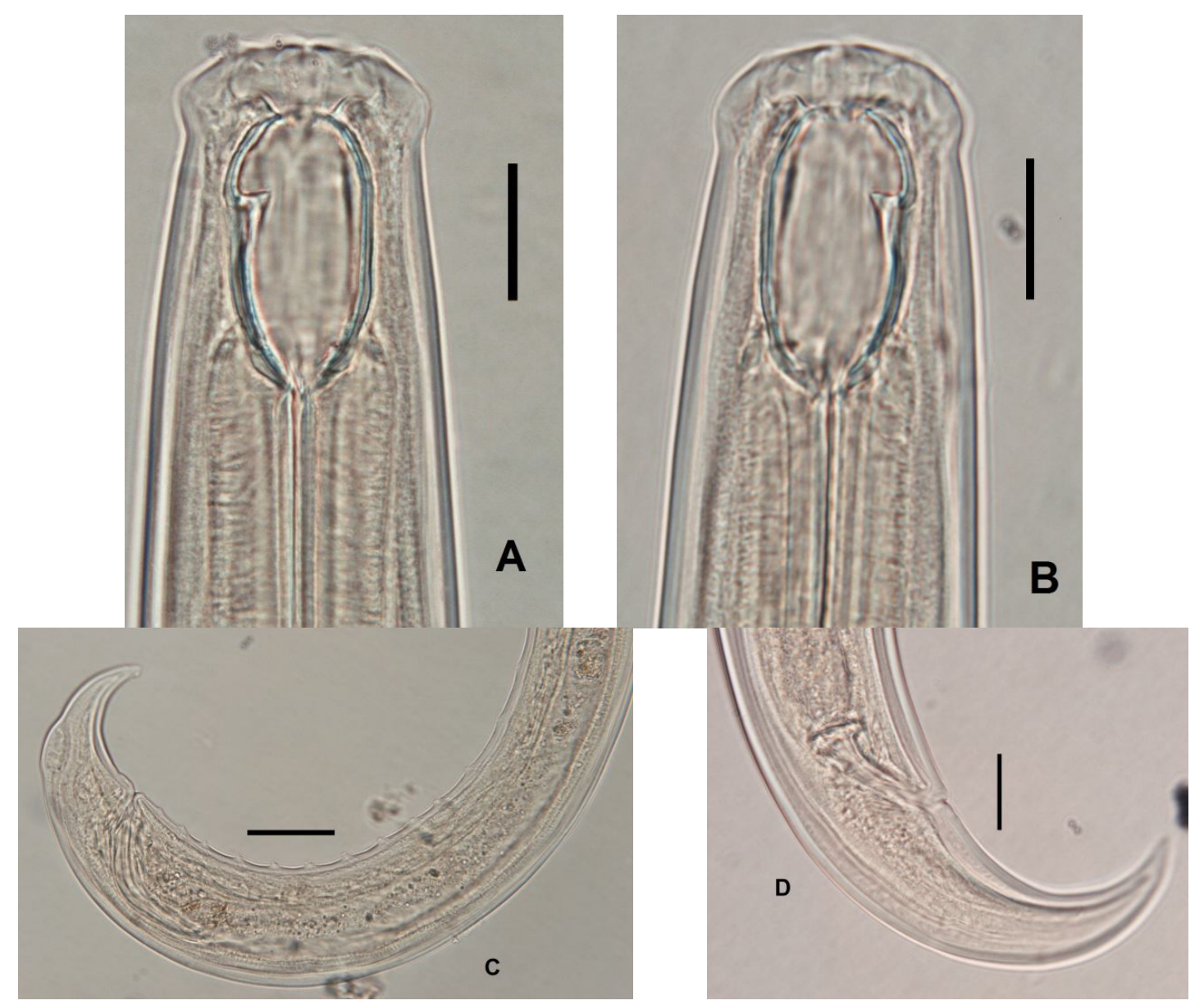

Figure 2. Coomansus venezolanus (Loof, 1964) Jairajpuri \& Khan, 1977

A: Female head region; B: Male head region; C, Male tail region; D, Femae tail region. Scale bars: A, B, $\mathrm{D}=20 \mu \mathrm{m}, \mathrm{C}=40 \mu \mathrm{m}$. Currently, two species of Coomansus have been recognized in Vietnam.

\section{REFERENCES}

1. Ahmad W., Jairajpuri M. S., 2010. Mononchida: The Predatory Soil Nematodes. In: Nematology Monographs and Perspectives 7. Eds. Hunt, D. \& Perry, R. Brill Leiden-Boston. 298pp.

2. Jairajpuri M. S., Khan W., 1977. Studies on Mononchida of India IX. Further division of the genus Clarkus Jairajpuri, 1970 with proposal of Coomansus n.gen. (Family Mononchidae Chitwood, 1937) and descriptions of two new species. Nematologica, 23(1): 89-96.

3. Jairajpuri M. S., Khan W., 1982. Predatory nematodes (Mononchida) with special reference to India. Associated Publishing Company. New Delhi, India. 131pp.

4. Loof P. A. A., 1964. Free living and plant parasitic nematodes from Venezuela. Nematologica, 10(2): 201-300.

5. Nguyen V. T., 2007. Fauna of Vietnam. Vol. 22. Free living nematodes: Mononhysterida, Araeolaimida, Chromadorida, Rhabditida, Enoplida, Mononchida, Dorylaimida. Science and Technics Publishing House, Ha Noi. 455 pp.

6. Seinhorst J. W., 1959. A rapid method for the transfer of nematodes from fixative to anhydrous glycerin. Nematologica, 4(1): 6769. 
7. Southey J. F., 1986. Laboratory methods for work with plant and soil nematodes. London: Her Majesty' Stationery Office. 202pp.

8. Vu Thi Thanh Tam, 2015. New records of five Iotonchus species (Mononchida, Iotonchidae) for Vietnam fauna and updated key to species from Vietnam. Tap chi Sinh hoc, 37(3): 265-271.
9. Vu Thi Thanh Tam, 2015. New recordsof predaceous nematodes Prionchulus (Mononchida, Mononchidae) from Bidoup-Nui Ba national park, Vietnam: 287-291. Proceedings of the $6^{\text {th }}$ national workshop on ecology and biological resources. October 2015. Science and Technics Publishing House, Ha Noi Vietnam.

\title{
GHI NHẬN MỚI LOÀI Coomansus venezolanus (Loof, 1964) Jairajpuri \& Khan, 1977 (Mononchida: Mononchidae) CHO KHU HỆ TUYẾN TRÙNG VIẸT NAM VỚI MÔ TẢ LẦN ĐẦU CÁ THỂ ĐỰC
}

\author{
Vũ Thị Thanh Tâm \\ Viện Sinh thái và Tài nguyên sinh vật, Viện Hàn lâm KH \& CN Việt Nam
}

\section{TÓM TÁtT}

Loài tuyến trùng Coomansus venezolanus (Loof, 1964) Jairajpuri \& Khan, 1977 được ghi nhận mới và mô tả cho khu hệ tuyến trùng Việt Nam. Con cái có kích thước cơ thể trung bình; dài 1,9-2,1 mm; xoang miệng được cutin hóa mạnh, đỉnh của răng lưng lớn nằm ở vị trí 26-30\% chiều dài của xoang miệng tính từ trên xuống; $\mathrm{V}=60-64 \%$. Một cá thể đực cũng đã lần đầu tiên được ghi nhận có 15 nhú sinh dục nằm cách đều nhau; spicule có chiều dài $74 \mu \mathrm{m}$, phần phụ bên dài $12,5 \mu \mathrm{m}$ và gubernaculum dài $11,4 \mu \mathrm{m}$. Số đo và các đặc điểm mô tả của quần thể tuyến trùng ở Việt Nam phù hợp hoàn toàn với số đo và mô tả của mẫu chuẩn ngoại trừ cơ thể hơi mảnh mai hơn $(\mathrm{a}=27-31$ so với $\mathrm{a}=21)$ và đuôi hơi ngắn hơn $(\mathrm{c}=20-28$ so với $\mathrm{c}=18)$. Số đo này cũng phù hợp với số đo của một cá thể khác được ghi nhận ở Himachal Pradesh, Ấn Độ.

Tì khóa: Coomansus, ghi nhận mới, tuyến trùng, Lạng Sơn, Việt Nam.

Received: 1 April 2016, accepted 20 September 2016 